\title{
Editorial - Novel approaches for improving safety, health benefits and sustainability of grain based foods
}

Improving the safety of food products and enhancing beneficial health effects is a necessity and a major challenge. In addition to well established practices, often laid down in ISO based systems and procedures for guaranteeing safety and a constant product quality, new approaches are explored in a wide range of research and development activities - triggered by, for instance, the preference for applying ingredients and processes considered as natural, the need for finding high value application of by-products and by many other factors from practice driven to curiosity driven ones. This issue of Quality Assurance and Safety of Crops \& Foods includes a set of papers showing a range of novel approaches, that also may contribute to limit wastes and increase food security and sustainability.

Exploring and exploiting beneficial effects of fermentation and other natural, biological processes is a key topic in this issue. Topics covered include the reduction of levels of acrylamide by dough fermentation, and of aflatoxin levels by dough fermentation and incubation of the contaminated material with kefir grains. Also in other recent studies - e.g. in the now completed EU HealthBread project (www.healthbread.eu) - fermentation - 'bio-processing' - has been identified as a key factor contributing to the quality of bread. Related to fermentation, controlled sprouting, discussed here in a review, is increasingly used as a natural process for producing high quality food products and additives for novel applications outside the malted beverage sector.

Another review article pays attention to the impact of dietary fibre and 'probiotic' bacteria on fermentation processes in the colon, its impact on composition of the gut microbiota and related health benefits, which is becoming a hot topic in food and health research.

The World Wildlife Fund's Livewell 2020 initiative presents as first guideline for 'Making a difference to our health and the planet: eat more plants - enjoy vegetables and wholegrains', thereby taking into account the low carbon footprint of vegetable foods in general and most grain based products in particular. It also recommends to substantially reduce the waste of food. Nutrition policy makers are starting to include sustainability in addition to human health in dietary guidelines. Papers in this issue explore and describe the use in foods of natural by-products for decontamination and enhancing anti-bacterial properties for prevention of spoilage during storage

Selection of optimal raw materials and ingredients and processing conditions for enhancing nutritional benefits is and remains an important topic in product development and is well represented in this issue. Blending of flour with immature wheat is presented as an option for increasing levels of beneficial nutrients. As indicated in a nutrition study with biscuits, sorghum as raw material may enhance satiety to a greater extent than wheat; communication of these results were honored with a Best Presentation award in ICC's $6^{\text {th }}$ International Dietary Fibre Conference (Paris, June 2015). Valuable information on the processing of rice on levels of ferulic acid - the main anti-oxidant in cereal grains - and levels of phytic acid is provided as well in this issue.

In summary, all these novel approaches combining a rational use of some raw materials and processes to guarantee safety and health of food products are opening the way for potential innovations. Moreover, as such approaches tend also to limit wastes and losses in the food sector, they could contribute to improve food security.

\section{Joel Abecassis and Jan Willem van der Kamp}

INRA, Montpellier, France

TNO Food and Nutrition, Zeist, the Netherlands 
